\title{
Transplantation of 3D scaffolds seeded with human embryonic stem cells: biological features of surrogate tissue and teratoma-forming potential
}

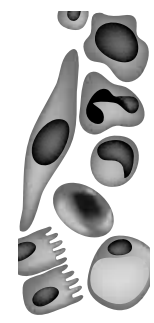

\author{
Justin G Lees', \\ Sue Anne Lim², \\ Tristan Croll2, \\ Georgia Williams ${ }^{1}$, \\ Sylvia Lui ${ }^{1}$, \\ Justin C ooper-W hite? \\ Leon R M CQ uade', \\ Bagyalakshmi \\ M athiyalagan ${ }^{1} \&$ \\ Bernard E Tuch ${ }^{1 \dagger}$
}

${ }^{\dagger}$ Author for correspondence 1 U niversity of $\mathrm{N}$ ew South Wales, D iabetes Transplant U nit, Prince of Wales H ospital, Sydney, Randwick, N SW 2031, Australia Tel.: +61 293824 814; Fax: +61 293824 826; E-mail: b.tuch@unsw.edu.au ${ }^{2} U$ niversity of Q ueenstand,

Tissue Engineering and M icrofluidics Laboratory, Division of Chemical Engineering and the Australian Institute for Bioengineering and N anotechnology, Brisbane Australia

Keywords 3D cell culturing human embryonic stem cells, scaffolds, surrogate tissue, teratomas

future, part of $_{\text {f }}$ SS

\begin{abstract}
Aim: To generate complex surrogate tissue by transplanting 3D scaffolds seeded with human embryonic stem cells ( $\mathrm{hESCS}$ ) between the liver lobules of severe combined immunodeficient (SCID) mice and to assess the teratoma-forming potential.

Materials \& methods: 3D poly-(lactic-co-glycolic acid) (PLGA) scaffolds coated with laminin were seeded with hESCs and then transplanted between the liver lobules of SCID mice. After a period of in vivo differentiation, the scaffolds were retrieved and analyzed using reverse transcription polymerase chain reaction, immunofluorescent staining and scanning electron microscopy. Results: A proportion of the hESCs within the scaffolds differentiated into cells that produced proteins characteristic of specific tissues, including endoderm and pancreatic markers glucogon-like peptide- 1 receptor, islet amyloid polypeptide and Insulin. Markers of hepatic and neuronal lineages were also investigated. Major matrix proteins abundant in multiple tissue types, induding collagen I, laminin and collagen IV, were found to be profuse within the scaffold pores. Transplantation of the seeded scaffolds between liver lobules also resulted in extensive vascularization both from host blood vessel incursion and the differentiation of hESCs into endothelial progenitor cells. An investigation of teratomaforming potential demonstrated that transplantation of 3D scaffolds seeded with hESCs will, under certain conditions, lead to the growth of teratomas. Discussion: Transplantation of 3D scaffolds seeded with hESCs between liver lobules resulted in the development of surrogate tissue containing cells that produced proteins representing the pancreatic, hepatic and neuronal lineages, the assembly of an extracellular matrix structure and the formation of a vasculature. hESCs seeded within 3D scaffolds and transplanted into SCID mice were capable of forming teratomas. However, the formation and progression of teratoma growth is shown to be dependant on both the site of transplantation and the treatment of cells prior to transplantation.
\end{abstract}

Regenerative medicine involves the development of surrogate tissue for the replacement of diseased or damaged tissues. Self renewal of human embryonic stem cells (hESCs) in a pluripotent state provides a unique source of cells for prospective therapies. hESC $s$ differentiated in a monolayer can be induced to exhibit characteristics of specific cell phenotypes, such as definitive endoderm progenitors and insulin-producing $\beta$-cells $[1,2]$. The differentiation strategies used to achieve such outcomes are based on the ontology of precursor cells and involve exposure to factors that are known to play key roles in tissue development. The responsiveness of hESC $s$ to this type of signaling indicates that efficient differentiation strategies must provide hESC s with factors that are encountered by target tissue progenitors during in vivo development [3].
Another important factor that is characteristic of in vivo tissue development is the formation of elaborate 3D structures. The structure of a tissue will, in most circumstances, incorporate a complex extracellular matrix (ECM) and an intricate vasculature. It has been demonstrated that the efficient function of multiple cell types, including islet hormone-producing cells, is dependent on matrix-producing and endothelial cells that provide a 3D support structure and sufficient vascularization [4-6]. It has also been demonstrated that islets do not function efficiently postisolation without restoration of an effective matrix structure [7]. Therefore, the 3D interactions between numerous cell types plays an important role in the function of tissue and may also be required for the efficient production of surrogate tissue structures derived from hESCs. Indeed, it has been 
observed that, during the process of $h E S C S$ monolayer differentiation towards the pancreatic lineage, many of the resulting hormoneproducing cells formed within multilayered regions [1]. M imicking the 3D nature of in vivo tissue development by using 3D cell culturing could provide the necessary environment for optimal production of surrogate tissues.

D ifferentiated hESC s can be seeded onto 3D scaffolds to allow for complex 3D cellular interaction and the development of higher-order structures [8]. 3D cell culturing of mouse ESCS ( $m E S C s)$ has been shown to promote the expression of multiple genes related to $\mathrm{mESC}$ differentiation [9]. hESCs differentiated within the 3D environment of bioengineered scaffolds are also known to form complex tissue structures containing cells derived from all three somatic germ lineages [10].

In these experiments, differentiated hESC s were seeded onto 3D scaffolds and transplanted into severe combined immunodeficient (SCID) mice between liver lobules. After a period of in vivo differentiation, the scaffolds were retrieved for analysis. Due to our interest in diabetes, the focus was on the detection of markers of developing pancreatic tissue, although we also investigated markers of the hepatic and neuronal lineages. An investigation of matrix structure and vasculatureformation, which are vital characteristics of nearly all tissues, was also performed.

A significant impediment to the use of $\mathrm{hESC} s$ in regenerative medicine is the possibility of teratoma formation [11]. Prior to regulatory approval of any clinical application, the possibility of teratoma formation must be eliminated. In these experiments, we also investigated the progression of teratoma formation in relation to the site of 3D scaffold transplantation and the effect of in vitro treatment of hESC s seeded onto 3D scaffolds.

\section{Materials \& methods}

hESC propagation \& differentiation

All reagents were obtained from Invitrogen ${ }^{T M}$ (Invitrogen, C arlsbad, CA, USA) [101], unless otherwise stated. The hESC line hES-03 (ES Cell International, Singapore) [102] and its derivative cell line EN VY [12] (M onash I mmunology and Stem Cell Laboratories, Australia) were maintained at $37^{\circ} \mathrm{C}, 5 \% \mathrm{CO}_{2}$ on top of a $\gamma$-irradiated ( $45 \mathrm{~Gy}$ ) human fetal fibroblast feeder layer. hESC colonies were grown in serumreplacement media (Knockout Dulbecco modified Eagle medium [D M EM ], 20\% KO-Serum
Replacement ${ }^{\circledR}, \quad 2 \mathrm{mM}$ L-glutamine, $0.1 \mathrm{mM}$ nonessential amino acids, $0.1 \mathrm{mM} \quad \beta$-mercaptoethanol, $1 \times$ insulin-transferrin-selenium, $25 \mathrm{U} / \mathrm{ml}$ penicillin and $25 \mu \mathrm{g} / \mathrm{ml}$ streptomycin). $4 \mathrm{ng} / \mathrm{ml}$ of fibroblast growth factor (FGF)-2 was added daily into the culture media. hESC colonies were propagated by enzymatic dissociation (0.05\% trypsin/0.076 g/l ethylenediaminetetraacetic acid) and transferred to newly irradiated feeder layers every 7 days.

\section{Differentiation}

Pluripotent colonies were exposed to collagenase IV and detached from the feeder layer. Colonies were dissociated and transferred to fibronectin-coated (Becton Dickinson, San Jose, CA, U SA) [103] six-well plates for 7 days differentiation, where they formed adherent monolayers in serum-replacement media prior to scaffold seeding. For most experiments, the media was supplemented with activin $A$ $(40 \mathrm{ng} / \mathrm{ml} ; R \& D$ systems, $M$ inneapolis, $M N$, USA) [104], epidermal growth factor (EGF; $100 \mathrm{ng} / \mathrm{ml} ; \quad R \& D$ systems) and FGF-2 (2 ng/ml). In additional experiments relating to the assessment of teratoma-forming potential, hESCs were also differentiated for 7 days as adherent monolayers in serum-replacement media without growth factor supplements, in serum-containing media (DMEM, 10\% fetal bovine serum, $2 \mathrm{mM}$ L-glutamine, $25 \mathrm{U} / \mathrm{ml}$ penicillin and $25 \mu \mathrm{g} / \mathrm{ml}$ streptomycin) and 5 days as suspension cultures in serum-replacement media prior to scaffold seeding.

Poly-(lactic-co-glycolic acid)

scaffold manufacture

The procedures for fabrication of 3D poly-(lactic-co-glycolic acid) (PLGA) scaffolds using the thermal-induced phase separation (TIPS) technique has been detailed elsewhere and are only described here briefly [13]. 5\% (w/v) PLGA was dissolved in 1,4-dioxane at $45^{\circ} \mathrm{C}$ for $2 \mathrm{~h}$ to make a homogenous solution. The PLGA-1,4-dioxane solution was then cast into $12 \times 35 \mathrm{~mm}$ glass vials for manufacture of the TIPS cylindrical disks. The cast PLGA-1,4-dioxane solutions were then quenched at $-18^{\circ} \mathrm{C}$ in a refrigerated cooling bath for at least $2 \mathrm{~h}$ to induce a solid-liquid phase separation. The solidified PLGA solution was subsequently vacuum dried under approximately $10^{-3} \mathrm{mbar}$ at $0^{\circ} \mathrm{C}$ for $24 \mathrm{~h}$ to remove the solvent. The scaffolds were then carefully removed from the mould, washed in ethanol, vacuum dried and stored in desiccators until 
needed. U sing a surgical blade, these scaffold cylinders of $12 \mathrm{~mm}$ diameter were tailored to havea width of approximately 1-2 $\mathrm{mm}$. The surface area of the scaffold was approximately $15 \mathrm{~mm}^{2} / \mathrm{mm}^{3}$.

\section{Seeding hESCS onto 3D scaffold}

Sterilized scaffolds were coated with $150 \mu \mathrm{g}$ of laminin (approximately $60 \mathrm{ng} / \mathrm{mm}^{2}$ of scaffold) in phosphatebuffered saline (PBS) for $1 \mathrm{~h}$. D ifferentiating $h E S C s$ growing in adherent monolayers were exposed to collagenase IV. The enzyme was removed before cells were washed and mechanically dissociated into small clusters. Cells from two wells of a six-well plate (approximately $1.2 \times 10^{6}$ cells) were transferred into each scaffold. Seeded scaffolds were cultured for at least 7 days in vitro in the same media that cells were exposed to prior to scaffold seeding. For assessment of teratoma-forming potential, certain scaffolds were coated with $200 \mu \mathrm{g}$ of fibronectin (approximately $80 \mathrm{ng} / \mathrm{mm}^{2}$ of scaffold) in PBS for $1 \mathrm{~h}$ and seeded with hESC clusters grown as suspension cultures in serum-replacement media. For assessment of teratoma-forming potential, seeded scaffolds were, in some circumstances, cultured in vitro for up to 5 weeks prior to transplantation in the same media that cells were exposed to prior to scaffold seeding.

\section{Scaffold transplantation \& retrieval}

Seeded scaffolds were transplanted separately into individual SCID mice between liver lobules for up to 7 weeks. Vehicle scaffolds, which did not contain hESCs, were transplanted into separate mice. In some circumstances, for assessment of teratoma-forming potential, a seeded scaffold was cut in half and each half was transplanted either between liver lobules or subcutaneously within the same individual mouse. In most cases, three seeded scaffolds were transplanted separately into mice for each condition tested. Two seeded scaffolds were transplanted for the 5-week in vitro experiments and one seeded scaffold was transplanted into each site for the comparison of subcutaneous and liver lobule teratoma progression. At specified time points, mice were killed and retrieved scaffolds were cut into portions to be used for analysis.

\section{Reverse transcription polymerase} chain reaction

Seeded scaffolds and adherent monolayers were exposed directly to lysis buffer, and messenger (m)RNA was isolated using a Quiagen spin column kit (H ilden, Germany) [105]. mRNA was converted to complementary D N A using Abgene Reversel T kit (Rochester, NY, USA) [106]. Polymerase chain reaction (PCR) was performed using an Abgene Redhot Taq kit. PCR was carried out for 35 cycles with an annealing temperature of $58^{\circ} \mathrm{C}$. The primers used are listed in Tablel

\section{Cell staining}

Pluripotent $h E S C$ colonies were stained with a Fuchsin Substrate-C hromogen System (D akoC ytomation) for detection of alkaline phosphatase activity. Formalin-fixed scaffold sections were stained with hematoxylin and eosin $(H \& E)$.

\section{Human fetal tissue}

Human fetal pancreatic tissue was obtained from terminated pregnancies with informed parental consent. Approval for the use of human fetal tissue was granted by the appropriate institutional human research ethics committee.

\section{Immunofluorescent staining}

Sixteen-week human fetal pancreatic tissue and retrieved scaffolds were fixed for at least $1 \mathrm{~h}$ in $10 \%$ neutral buffered formalin before being embedded in paraffin. Sections were cut onto silane-coated slides, dewaxed and rehydrated. Scaffold sections were then treated with Reveal ${ }^{\circledR}$ (Biocare medical, Walnut creek, CA, USA) [107] for $20 \mathrm{~min}$ at $90-95^{\circ} \mathrm{C}$. Imagel T FX was then applied for $30 \mathrm{~min}$ at room temperature to block nonspecific binding. Pluripotent hESC colonies growing on cover slips were fixed in PBS containing 4\% paraformaldyhyde dissolved in PBS and blocked in PBS containing 10\% normal donkey serum plus $0.1 \%$ triton- $x-100$. Primary and appropriate secondary antibodies were applied and cells were then stained with 4,6-diamidino-2-phenylindole. Primary antibodies used were: mouse antihuman/mouse collagen I (1:50; Chemicon, Temecula, CA, U SA) [108]; goat antihuman/mouse collagen IV (1:20; Chemicon); rabbit antihuman/mouse laminin (1:50; Chemicon); mouse antihuman mitochondria-specific antibody (1:30; Chemicon); mouse antihuman stage-specific embryonic antigen (SSEA)-4 (1:100; Chemicon); rabbit antihuman/mouse islet amyloid polypeptide (IAPP; 1:100; Beta Cell Biology Consortium, antibody core); mouse antihuman/mouse glucogon-like peptide (GLP)-1 (1:50; kind gift from D Drucker); guinea pig antihuman/mouse insulin (1:1000; D ako ytomation); rabbit antihuman $\alpha$-fetoprotein (AFP; 1:20; Sigma, St Louis) [109]; mouse 
Table 1. Primer sequences used for polymerase chain reactions.

\begin{tabular}{llll} 
Gene & Forward & Reverse & Size \\
INS & GCCTTGTGAACCAACACCTG & TCCACAATGCCACGCTCT & 209 \\
PDX1 & CCCATGGATGAAGTCTACC & GTCCTCCTCCTITCCAC & 262 \\
\hline NEUROG3 & TGGCACTGAGCAAGCAG & TGGGAGACTGGGGAGTAGAG & 311 \\
\hline SOX17 & AGCGCCCTCACGTGTACTA & CTTCACACGCCGTGCAGAT & 246 \\
\hline GATA6 & CCATGACTCCAACTCCACC & ACGGAGGACGTGACTTCGGC & 214 \\
AFP & AAAAGCCCACTCCAGCATC & GCAGACAATCCAGCACATCTC & 450 \\
\hline BMP4 & GGAGGAGGAGGAAGAGCAGAT & GGTTGGTGAGTGAGGTGGT & 785 \\
NES & GGCAGCGTTGAACAGAGGTTG & CTCTAAACTGGAGTGGTCAGGGCT & 718 \\
\hline OCT4 & ATTCAGCCAAACGACCATCT & CAGCAGCCTCAAAATCCTCT & 371 \\
ACTB & ACGGCATCGTCACCAACT & AGGAAGGAAGGCTGGAAGAG & 583 \\
\hline
\end{tabular}

ACTB: $\beta$-actin; AFP: $\alpha$-fetoprotein; BMP4: Bone morphogenic protein 4; GATA6: GATA-binding protein 6; INS: Insulin; NES: Nestin; NEUROG3: Neurogenin 3; OCT4: Octamer-binding transcription factor 4; PDX1: Pancreatic and duodenal homeobox 1; SOX17: Sex-determining region Y-box 17.

antihuman nestin (1:200; Chemicon); mouse antihuman $\beta$-III tubulin (1:200; Sigma); mouse antihuman CD 34 (1:50; C hemicon); and rabbit antihuman CD 31 (1:50; Santa Cruz, Santa Cruz, CA, USA) [110]. Slides were viewed using a Zeiss Axio-flop II fluorescent microscope with an Axiocam attached. Q uantification of positive cell staining was performed by counting from four separate regions of scaffold sections and at least 1000 separate nuclei.

Scanning electron microscopy

Seeded scaffolds were fixed and dehydrated through a graded ethanol series $(50,75,85,95$, $100 \% \times 2 ; 10 \mathrm{~min}$ in each). The scaffolds were then chemically dried using hexamethyldisilazane (Sigma) and gold sputter coated in an EmiTech K550x (Kent, England) [111]. Samples were examined in a Cambridge Stereoscan 360 operated at $20 \mathrm{kV}$.

\section{Results}

Pluripotent hESC colonies were maintained on top of a fibroblast feeder layer using a standard propagation technique [14]. Under these conditions, the hESC s stained positively for the pluripotency markers alkaline phosphatase (Figure1A) and SSEA-4 (Figure1B). Pluripotent hESC s were dissociated from the feeder layer and initially transferred to an adherent monolayer culture.

Porous customized scaffolds were cut from 3D PLGA tubes manufactured using the TIPS technique $(12 \mathrm{~mm}$ diameter and $1-2 \mathrm{~mm}$ in width) [13]. The scaffolds were coated with laminin to improve the efficiency of cellular adhesion. The scaffolds, which contained pores that ranged in size up to approximately $300 \mu \mathrm{m}$ in diameter (Figre 1C), were manufactured to form a complex microenvironment (Figure ID). The scaffolds were seeded with differentiating hESC $s$ and cultured in vitro. A scanning electron microscope (SEM) image shows cells adhered to the internal surfaces of the scaffold (Figre1E). $\mathrm{H}$ istological staining through the center section of a scaffold demonstrated that the cells were capable of forming complex structures in vitro within the scaffold (Figure 1F).

Seeded scaffolds were transplanted between liver lobules of SCID mice for in vivo differentiation. Vehicle scaffolds, not seeded with hESC , were also transplanted and subsequently found to be populated by host mouse cells. Human mitochondria-specific antibody identified cells of human origin. After 1 week in vivo, there is a clear distinction between human cells at the edge of the scaffold and mouse liver cells (Figure1G). A section of a scaffold retrieved after 5 weeks demonstrates that most cells within the scaffold were of human origin (Figre1H).

Reverse transcription (RT)-PCR analysis of the adherent monolayer and retrieved seeded scaffolds demonstrated the expression of endoderm lineage markers SOX17 and GATA6. NEUROG3, an essential transcription factor for endocrine specification in the developing pancreas, was detected throughout the procedure. The pivotal pancreatic transcription factor PDX1 and the $\beta$-cell regulatory hormone IN S (insulin) were both detected after 5 weeks in vivo differentiation. AFP, a marker associated with both the extra-embryonic and hepatic endoderm lineages, was detected posttransplantation. Furthermore, bone morphogenetic protein 4 (BM P4), a marker of progenitors from the mesoderm lineage and NES (nestin), a 
Figure 1. 3D cell culturing and transplantation procedure.
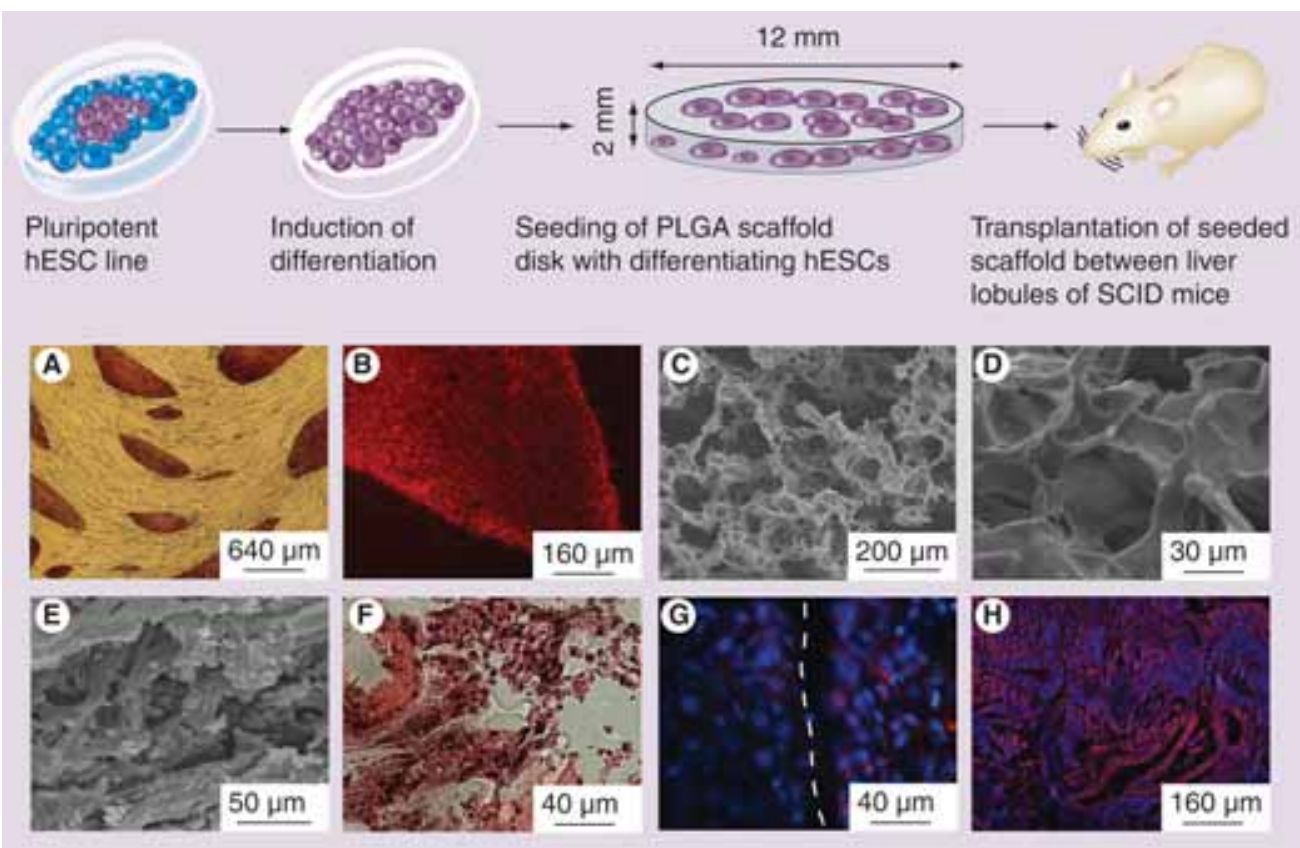

(A) Alkaline phosphatase staining of hESC colonies. (B) Stage-specific embryonic antigen-4 immunostaining of hESC colony. (C-D) Scanning electron microscopy (SEM) images of unseeded PLGA scaffolds. (E) SEM image of seeded scaffold after 1 week in vitro. (F) Hematoxylin and eosin-stained section of seeded scaffold after 1 week in vitro. Immunostaining of seeded scaffolds retrieved after (G) 1 week in vivo, human mitochondria-specific antibody (HM S; red) and (H) 5 weeks in vivo, HM S (red).

hESC: Human embryonic stem cell; PLGA: Poly-(lactic-co-glycolic acid); SCID: Severe combined immunodeficient.

marker of progenitors from the ectoderm lineage, were detected throughout the period of in vivo differentiation (Figre2).

Immunofluorescent analysis of the hESCseeded scaffolds demonstrated that the differentiated cells produced proteins characteristic of multiple lineages. Positive staining within the scaffold for GLP-1 receptor was observed (Figre2A). The ligand for this receptor (GLP-1) is implicated in the development of pancreatic progenitors [15]. Isolated clusters of cells were also detected staining for IAPP (Figre2B) and for insulin (Figre2C). As expected, these proteins are readily detectable in developing pancreatic tissue (Figure2D-F). The extra-embryonic and hepatic marker AFP was detected in what appeared to be sheets of cells (Figure2G). D ense clusters within the scaffold were also found to contain cells that produced substantial quantities of neuronal markers, including nestin (Figure2H) and $\beta$-III tubulin (Figre21). Quantification of the percentage of positively stained cells revealed that insulin was detected in $2.7 \%$ of cells, AFP was detected in $2.9 \%$ of cells and the neuronal marker nestin was detected in $38 \%$ of cells (Figre2 \& Table2).
Further immunofluorescent staining of retrieved seeded scaffolds demonstrated the presence of specific ECM proteins. M ost of the pore space was shown to stain positively for collagen I (Figre3A), laminin (Figure3B) and collagen IV (Figure3C). The laminin staining is more dispersed and complex in comparison to the surface staining pattern observed from nonseeded, nontransplanted laminin-coated scaffolds (data not shown). Therefore, excess laminin was produced within the scaffold during the in vivo period. W ithin retrieved scaffolds, SEM imaging of the cellular niche topography revealed abundant fibrous structures that represent the matrix network (Figre3G). These ECM proteins are shown to be profuse in developing tissues, such as the pancreas (Figre3D-F).

Vascularization over the scaffold surface was visible after 1 week in vivo (Figure3) and SEM imaging depicted mouse host blood vessels invading the scaffold (Figure3H). $\mathrm{H} \& \mathrm{E}$ staining identified blood vessels containing red blood cells (Figure3K). After 5 weeks in vivo, a proportion of the hESC $s$ within scaffolds were found to stain for the endothelial markers CD 34 and 


\section{Figure 2. Reverse transriptase polymerase chain reaction analysis and immunofluorescent analysis of in vitro and in vivo differentiation.}
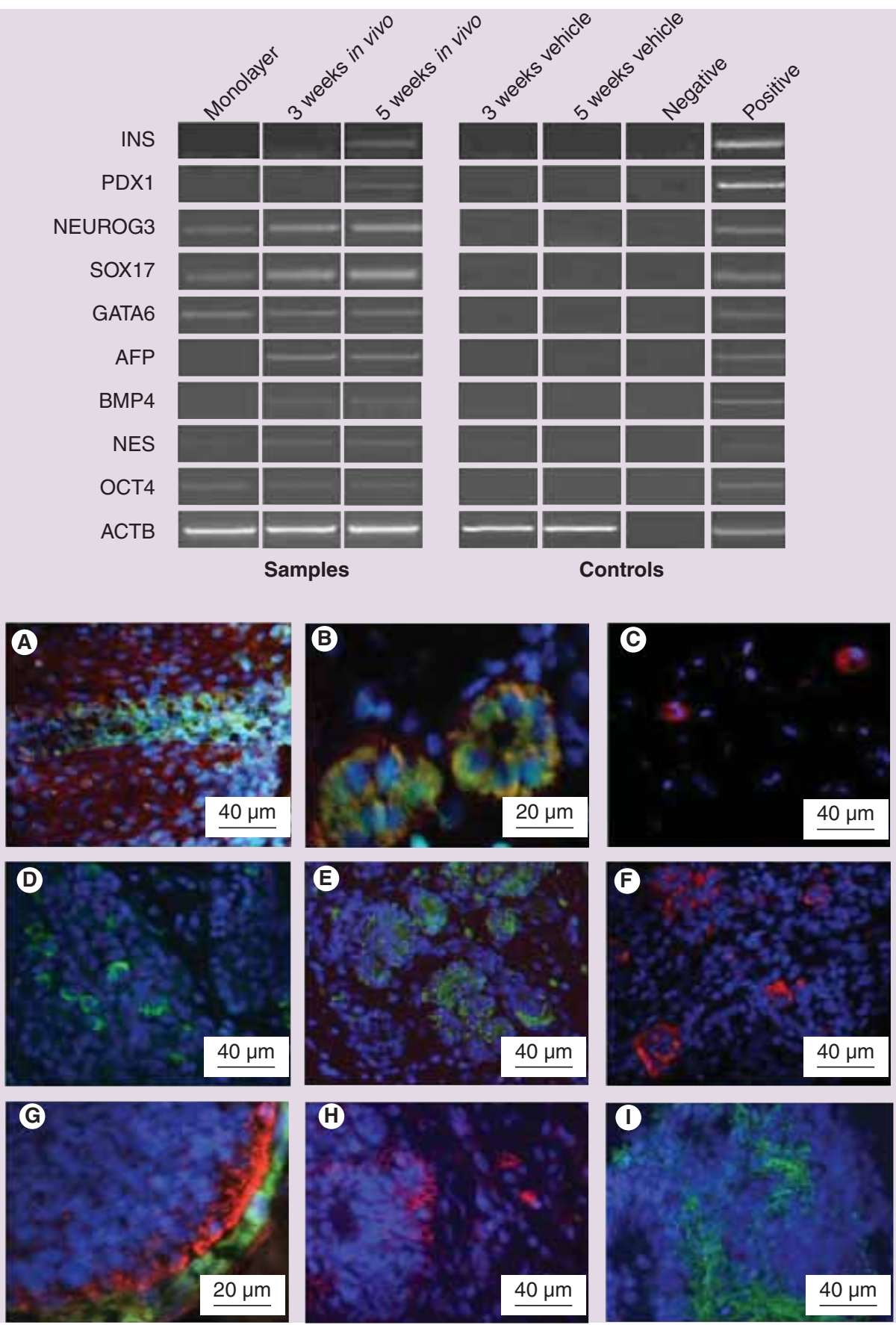

Reverse transcription polymerase chain reaction (RT-PCR) results for human embryonic stem cells (hESCs) at different stages of the differentiation procedure. Positive control used was human fetal pancreas, except for OCT4, which was pluripotent hESC. Vehicle scaffolds and $\mathrm{H}_{2} \mathrm{O}$ were used as negative controls. Immunostaining of hESC-seeded scaffolds retrieved after 5 weeks in vivo: (A) glucogon-like peptide (GLP)-1 receptor (green) and human mitochondria-specific antibody (HMS; red); (B) Islet amyloid polypeptide (IAPP; green) and HM S (red); (C) insulin (red). 16 weeks human fetal pancreas: (D) GLP-1 receptor (green); (E) IAPP (green); (F) insulin (red). Immunostaining of hESC-seeded scaffolds retrieved after 5 weeks in vivo: (G) AFP (green) and HMS (red); (H) nestin (red); and (I) $\beta$-III tubulin (green).

ACTB: $\beta$-actin; AFP: $\alpha$-fetoprotein; BM P4: Bone morphogenic protein 4; GATA6: GATA-binding protein 6 ; INS: Insulin; NES: Nestin; NEUROG3: Neurogenin 3; OCT4: Octamer-binding transcription factor 4; PDX1: Pancreatic and duodenal homeobox 1; SOX17: Sex-determining region Y-box 17. 


\begin{tabular}{|c|c|c|c|}
\hline & Insulin & $\alpha$-fetoprotein & Nestin \\
\hline Positive* (\%) & 2.7 & 2.9 & 38 \\
\hline Standard error & 1.7 & 1.3 & 5.6 \\
\hline
\end{tabular}

*Percentage of cells staining positive for indicated protein.

CD 31 (Figure31). The CD 34 antibody is human specific and did not stain control sections of mouse pancreas or liver (data not shown). There fore, the CD 34-positive cells in the scaffold are hESC progeny. CD 34- and CD31-positive endothelial progenitors are plentiful in many developing tissues, as demonstrated by staining of the developing pancreas (Figre3L).

In vivo differentiation of hESCs on 3D scaffolds promoted lineage-specific protein production, ECM assembly and scaffold vascularization but also involved the risk of teratoma formation. For the purposes of investigating teratoma-forming potential, both GFP-expressing and parental hESC s were used in separate experiments. We observed no difference in teratoma-forming capability between GFP-expressing and nonGFP-expressing hESC s. As expected, no teratomas were observed in mice transplanted with vehicle control scaffolds (Figre4A). H owever, transplantation between liver lobules of hESC $s$ seeded onto laminin-coated scaffolds that were differentiated in vitro for 2 weeks in growth factor-supplemented serum-replacement media resulted in the formation of teratomas (Figure4B). Interestingly, seeded scaffolds treated in the exact same way and transplanted subcutaneously produced distinctly smaller teratomas (Figre4C). hESC $s$ were then seeded onto laminin-coated scaffolds and differentiated in vitro for 2 weeks in serum-replacement media without growth factor supplementation prior to transplantation between liver lobules. Removal of the growth factors combined with the 2-week in vitro differentiation period did not prevent the formation of teratomas (Figure4D). hESC s were also seeded onto laminin-coated scaffolds and differentiated in vitro for 2 weeks in serum-containing media without growth factor supplementation prior to transplantation between liver lobules. The use of serum-containing media and 2 weeks in vitro differentiation also resulted in teratoma formation (Figre4E). hESC S were then seeded onto laminin-coated scaffolds and differentiated in vitro for 5 weeks in growth factor-supplemented serum-replacement media prior to transplantation between liver lobules. This extended in vitro differentiation in the presence of growth factors did not inhibit the formation of teratomas (Figure4F). Finally, factors that might be prolonging pluripotency were identified and substituted. hESCs were grown in serumreplacement media without growth factor supplementation as suspended clusters and seeded into fibronectin coated scaffolds. These seeded scaffolds were cultured in serum replacement media without growth factor supplementation for 5 weeks in vitro. The GFP-expressing hESCs were identified in vitro throughout the entire scaffold using an overhead fluorescent microscope (Figure4G). At higher magnification, it was possible to identify cells adhered to the surface of the scaffold (Figre4H). After 5 weeks in vitro, the seeded scaffolds were transplanted between mouse liver lobules. In contrast to the other treatments, formation of teratomas was not observed (Figre4l).

\section{Discussion}

hESC $s$ differentiated in a monolayer and seeded onto laminin-coated 3D scaffolds formed complex 3D cellular structures that were subsequently transplanted into SCID mice for a period of in vivo differentiation. U pon retrieval, the scaffolds were assessed for biological features associated with specific tissues, including the production of proteins representing the pancreatic, hepatic and neuronal lineages, the assembly of an ECM structure and the formation of a vasculature. A further investigation was made into the teratomaforming potential of hESC-seeded scaffolds.

Serum-replacement media was used during the scaffold-seeding procedure in most experiments. hESC adhesion to the surface of PLGA scaffolds is restricted in serum-replacement media and ECM surface coatings are required to increase adhesion efficiency. For most experiments, laminin was selected to surface coat PLGA scaffolds prior to seeding. Previous studies have reported the use of $M$ atrigel ${ }^{\mathrm{TM}}$ (Becton Dickinson, San Jose, CA, USA), which contains laminin, to embed hESC $s$ in a gel within 3D scaffolds [10]. H owever, embedding cells in gel is technically distinct from coating the scaffold surface to induce cellular adhesion. The hESCs seeded onto laminin-coated scaffolds formed complex tissue structures in vitro within the scaffold. Fibronectin was also used to coat the scaffolds in some experiments relating to teratoma-forming potential and resulted in the adhesion of differentiated hESC $s$ to the surface of the scaffold. 
Figure 3. Immunostaining of human embryonic stem cell-seeded scaffolds retrieved after 5 weeks in vivo.
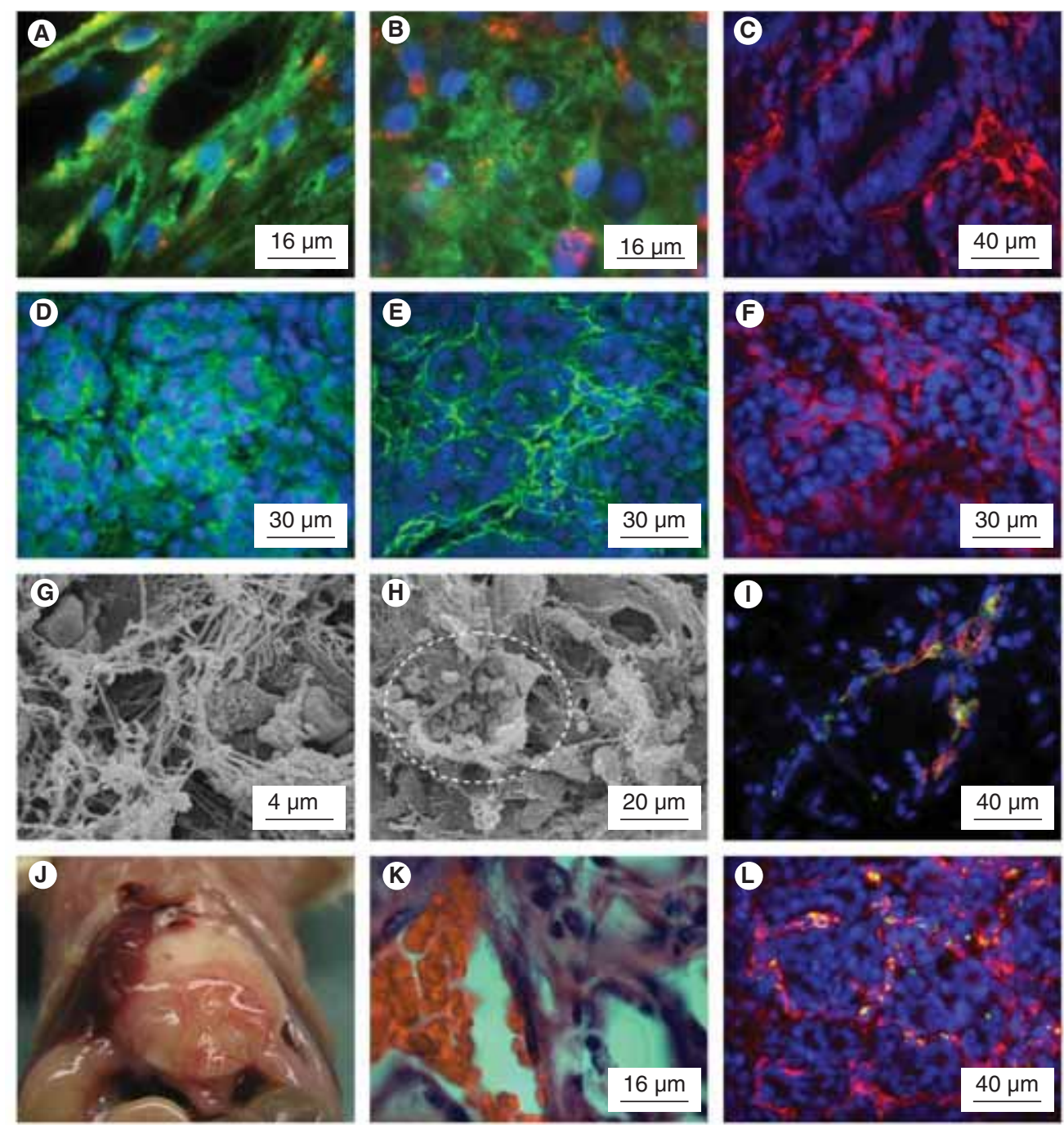

(A) Collagen I (green) and human mitochondria-specific antibody (HM S; red). (B) Laminin (green) and HMS (red). (C) Collagen IV (red). 16 weeks human fetal pancreas: (D) collagen I (green); (E) laminin (green); (F) collagen IV (red). Scanning electron microscopy (SEM) image of human embryonic stem cells (hESCs) seeded scaffolds retrieved after 1 week in vivo: (G) showing fibrous matrix and $(\mathbf{H})$ host blood vessel incursion; (J) Digital photo of vascularized scaffold after 1 week in vivo; (K) Hematoxylin and eosin-stained section of scaffold retrieved after 1 week in vivo. Immunostaining of hESC seeded scaffold retrieved after 5 weeks in vivo: (I) CD34 (red) and CD31 (green). Immunostaining of 16 weeks human fetal pancreas: (L) CD34 (red) and CD31 (green).

3D cell culturing and in vivo differentiation resulted in the detection of markers of various tissues. 0 ur research is primarily focused on developing a cellular-replacement therapy to treat Type 1 diabetes and, therefore, several pancreatic makers were investigated. The results demonstrated PD XI and IN S gene expression and cellular staining for GLP-1 receptor, IAPP and insulin. These results indicate the presence of cells with characteristics of pancreatic endocrine tissue, but the number of cells staining positive for insulin $(2.7 \%)$ was a small proportion of the total cell population. Investigation of other lineages indicated the presence of a small proportion of AFP-positive cells (2.9\%). AFP is detected in vivo in early extra-embryonic endoderm line age, although the production of AFP observed within the scaffold more likely represents the hepatic lineage because AFP gene expression was not detected during the in vitro differentiation 
Figure 4. Teratoma-forming potential of transplanted scaffolds.
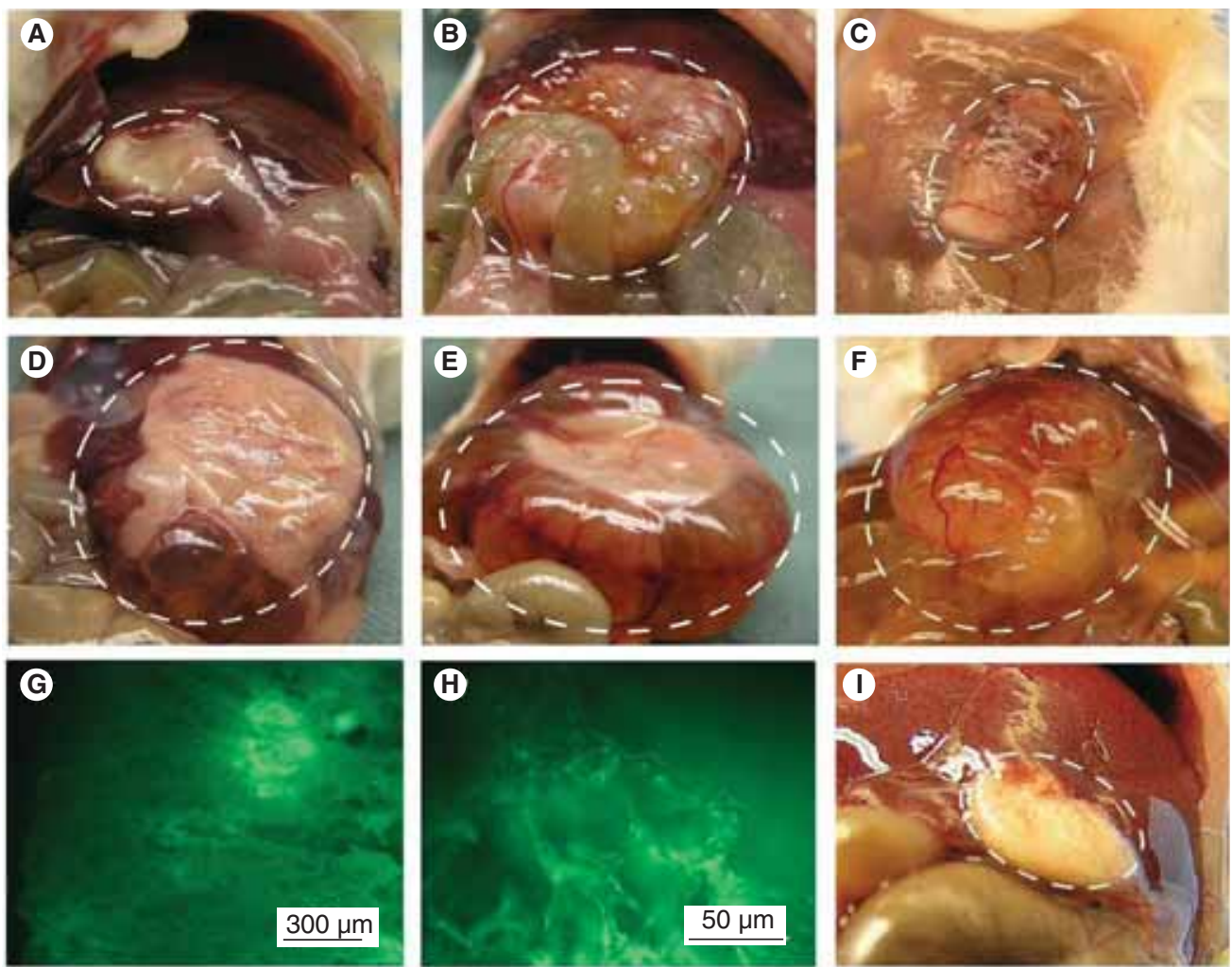

(A) Vehicle scaffold. (B) 2 weeks in vitro grow th factor treatment, seeded onto a laminin-coated scaffold and transplanted between liver lobules. (C) 2 weeks in vitro growth factor treatment, seeded onto a laminincoated scaffold and transplanted subcutaneously. (D) 2 weeks in vitro in serum-replacement media without growth factors, seeded onto a laminin-coated scaffold and transplanted between liver lobules. (E) 2 weeks in vitro in serum-containing media without growth factors, seeded onto a laminin-coated scaffold and transplanted between liver lobules. (F) 5 weeks in vitro growth factor treatment, seeded onto a laminincoated scaffold and transplanted between liver lobules. (G) Low magnification and $(\mathbf{H})$ high magnification images of green fluorescent protein-expressing human embryonic stem cell seeded onto scaffold prior to transplantation. (I) 5 weeks in vitro serum replacement without growth factors, seeded onto fibronectin coated scaffolds and transplanted between liver lobules.

period. A substantial proportion of the cells within scaffolds stained positive for the neuroectoderm lineage marker nestin (38\%). The neuroectoderm lineage is thought to be the default pathway for hESC differentiation [16], particularly in circumstances where cells are exposed to media containing high concentrations of phosphoinositide-3 kinase (PI3K) pathway agonists, such as insulin and insulin-like growth factor-1, which inhibit the development of the definitive endoderm lineage [2].

To achieve our goal of developing a Type 1 diabetes therapy using 3D culturing of hESCs, significant modifications and improvements are required. Future experiments will incorporate a differentiation strategy that exposes pluripotent $\mathrm{hESC}$ s to high concentrations of activin $\mathrm{A}$ in the presence of low serum [1] or a PI3K inhibitor [2] to produce definitive endoderm and the subse quent addition of retinoic acid [17] or the inhibition of sonic hedgehog [1] to generate pancreatic endocrine progenitors. The implementation of these and other yet to be elucidated procedures could produce a virtually homogenous pancreatic endocrine progenitor population for scaffold seeding. The seeded scaffolds could then be exposed to further differentiation factors in vitro, such as the incretin GLP-1 [18]. Cumulatively, these measures would likely be beneficial for the production of functional surrogate islet-like tissue containing a substantial proportion of insulin-producing cells. The resulting seeded scaffolds could then be assessed for the presence of a range of islet hormones, glucose-responsive insulin release, in vivo secretion of C-peptide and reversal of hyperglycemia in diabetic mice. 
However, it is unclear whether or not it would be optimal to seed 3D scaffolds solely with progenitors that represent the phenotype of the cells damaged in the tissue that is targeted to be replaced. It is possible that co-transplantation of other types of cells that produce auxiliary components of tissue, including the ECM and vasculature, will be required [8]. The ECM aids development of functional surrogate tissue because the ECM is involved in multiple aspects of cell metabolism, including adhesion, proliferation and differentiation [19]. Construction of an ECM supports cellular expansion into the 3D pore space of scaffolds [20]. Unlike monolayer cell culturing, 3D cell culturing promotes the production of complex ECM structures [21]. Specific ECM proteins, including laminin, collagen I and collagen IV, were detected in vivo within the 3D scaffolds. The staining of collagen IV also indicates the formation of a basement membrane structure. The presence of ECM proteins within the scaffold structure raises questions regarding their importance in terms of developing functional surrogate tissue. For example, it might be necessary to induce a portion of cells seeded onto 3D scaffolds to construct an ECM resembling the architecture of the tissue targeted for replacement [22]. If this were necessary, it would pose a significant bioengineering challenge due to the intricate arrangement of ECM proteins found within developing tissues, as demonstrated by staining of the developing pancreas [5].

Vascularization occurred in conjunction with in vivo differentiation of the transplanted cells. Transplantation of the scaffolds between liver lobules of the mice resulted in rapid invasion of the scaffold structure by the host vasculature. Extensive vascularization is an essential factor for the efficient function of surrogate tissue and the liver lobules could be considered as a beneficial site for scaffold transplantation. Similar to Levenberg and colleagues, we detected a proportion of the hESC s differentiating in vivo into endothelial progenitor cells [10]. If a surrogate vasculature was required in vitro, hESC $s$ could be deliberately differentiated towards an endothelial phenotype. The CD 34 and CD 31 endothelial progenitors, isolated from an enriched population using sorting techniques, could then be simultaneously seeded onto the scaffolds with target tissue progenitor cells and perhaps matrix-producing cells. Therefore, multiple cell types would be derived from the same hESC line using alternative directed differentiation protocols and seeded onto a communal scaffold.

Transplantation of developing tissue from first trimester fetuses can result in the formation of teratomas similar to that seen with the transplantation of pluripotent hESC s [23]. This occurrence could have a detrimental effect on the adoption of future hESC-derived cellreplacement therapies. Remnant pluripotent cells will result in teratomas and attempts to differentiate ESC s into functional tissue have been hampered by this occurrence [11]. We have examined teratoma formation resulting from the transplantation of hESC s seeded onto 3D scaffolds. Both the method of cell differentiation and the duration of in vitro treatment are relevant. In most of our experiments, the differentiation media we used was supplemented with growth factors that have conflicting activities depending of the type of media they are added to and the culturing environment in which they are used [24-26]. Both activin $A$ and basic FGF can reportedly maintain a pluripotent phenotype without a feeder layer or conditioned media $[25,27]$. This would have contributed to the persistence of pluripotent cells within the cell population seeded onto the scaffolds. Another factor that would have contributed to the presence of pluripotent cells within scaffolds post transplantation is the laminin surface coating because laminin is highly concentrated in early embryonic tissue and pluripotent $h E S C s$ express integrins that have laminin-binding capabilities [28].

When cells were transplanted onto fibronectin-coated scaffolds and exposed to extended in vitro differentiation in serum-replacement media not supplemented with growth factors, we did not observe teratoma formation. Therefore, the media composition and the scaffold ECM protein coating utilized must not promote a pluripotent phenotype. An investigation of the consequences of particular ECM protein coatings in relation to the adhesion of pluripotent hESC $s$ to PLGA surfaces is the subject of ongoing experiments [ $\mathrm{G}$ ao et al. Unpublished $\mathrm{D}$ ata].

Furthermore, in order to eradicate teratoma formation, the period of in vitro differentiation has to be sufficient to allow for all hESC s to differentiate beyond the pluripotent phenotype. Pluripotent cell populations do not immediately downregulate pluripotency properties once differentiation is initiated and, regardless of the environment, a certain period 
of differentiation is required. In our experiments, this period was always greater than 2 weeks.

This is the first reported transplantation of hESC-seeded scaffolds between liver lobules. While the liver is an excellent location for promoting rapid vascularization of transplanted scaffolds, it has recently been implicated in the promotion of teratoma growth [29]. O ur results agree with this observation, as demonstrated by the large variation in the size of teratoma growth observed between scaffolds transplanted between the liver lobules or subcutaneously. H owever, this should not deter transplantation of hESC -seeded scaffolds between liver lobules. Conversely, it is a beneficial site for transplantation because it will indicate rapidly whether the transplanted material contains remnant pluripotent cells capable of teratoma formation.

\section{Executive summary}

- Differentiating human embryonic stem cells (hESCs) seeded into 3D poly-(lactic-co-glycolic acid) scaffolds and transplanted between liver lobules were found to produce proteins that are characteristic of several tissues, including the pancreatic, hepatic and neuronal lineages.

- Matrix proteins that are abundant in multiple tissues, including collagen I, laminin and collagen IV, were detected within seeded scaffold pores.

- Transplantation of seeded scaffolds between the liver lobules resulted in extensive vascularization derived from both host blood vessel incursion and the differentiation of hESCs into endothelial progenitor cells.

- Transplantation of hESCs seeded onto 3D scaffolds under certain conditions led to the growth of teratomas, although the formation and progression of teratoma growth is shown to be dependant on both the site of transplantation and the treatment of cells prior to transplantation.

\section{Conclusion}

hESC s seeded onto 3D scaffolds and transplanted into SCID mice were shown to be capable of producing proteins characteristic of multiple lineages, including the pancreatic markers GLP-1 receptor, IAPP and insulin, the hepatic and extra embryonic marker AFP and the neuronal marker nestin. A complex ECM containing laminin, collagen IV and collagen I was also assembled within the scaffold pores. Furthermore, hESC $s$ differentiated into CD 34-positive endothelial progenitors within the scaffold, which werefound to be present along with an extensive host vasculature.

Remnant pluripotent cells remaining within the scaffolds prior to transplantation led to the formation of teratomas. Certain media supplements and the laminin coating of scaffolds would have contributed to the maintenance of pluripotent cells within the transplanted scaffolds. These factors could either be excluded from differentiation protocols or used at a time when the differentiating cell population no longer contains pluripotent cells. Even when pluripotency-prolonging factors are excluded, teratoma formation may still occur if the period of in vitro differentiation is restricted.

\section{Acknowledgements}

We gratefully acknowledge the technical assistance of $\mathrm{MS}$ Jenny N orman and M S M argaret Budanovic at the U N SW Electron M icroscopy Unit and the Prince of Wales Hospital SEALS for paraffin embedding and use of gamma irradiation source. The authors also acknowledge the financial support of the Juvenile Diabetes Research Foundation (JDRF) Beta Cell Alliance, Rebecca L Cooper Medical Research Foundation and the ARC D iscovery $G$ rant scheme (utilized in the production and design of scaffolds).

\section{Bibliography}

1. D'Amour KA, Bang AG, Eliazer $S$ et al.: Production of pancreatic hormone-expressing endocrine cells from human embryonic stem cells. Nat. Biotechnol. 24(11), 1392-1401 (2006).

2. $M C L$ ean $A B, D$ 'Amour $K A$, Jones $K L$ et al.: Activin a efficiently specifies definitive endoderm from human embryonic stem cells only when phosphatidylinositol 3-kinase signaling is suppressed. Stem Cells 25(1), 29-38 (2007).

3. Lees JG, Tuch BE: Conversion of embryonic stem cells into pancreatic $\beta$-cell surrogates guided by ontogeny. Regenerative M ed. 1(3), 327-336 (2006).

4. Konstantinova I, Lammert E: M icrovascular development: learning from pancreatic islets. Bioessays 26(10), 1069-1075 (2004).
5. Wang R, Li J, LyteK, Yashpal N K, Fellows F, Goodyer CG : Role for $\beta 1$ integrin and its associated $\alpha 3, \alpha 5$, and $\alpha 6$ subunits in development of the human fetal pancreas. Diabetes 54(7), 2080-2089 (2005).

6. H ammar E, Parnaud G, Bosco D et al.: Extracellular matrix protects pancreatic $\beta$-cells against apoptosis: role of short- and long-term signaling pathways. D iabetes53(8), 2034-2041 (2004).

7. Wang RN, Rosenberg L: M aintenance of $\beta$-cell function and survival following islet isolation requires re-establishment of the islet-matrix relationship. J. Endocrinol. 163(2), 181-190 (1999).

8. Levenberg S, Rouwkema J, M acdonald M et al.: Engineering vascularized skeletal muscle tissue. N at. Biotechnol. 23(7), 879-884 (2005).
9. Liu H, Lin J, Roy K: Effect of 3D scaffold and dynamic culture condition on the global gene expression profile of mouse embryonic stem cells. Biomaterials 27, 5978-5989 (2006).

10. Levenberg S, H uang N F, Lavik E, Rogers AB, Itskovitz-Eldor J, Langer R: D ifferentiation of human embryonic stem cells on three dimensional polymer scaffolds. Proc. N atl Acad. Sci. U SA 100(22), 12741-12746 (2003).

11. Fujikawa T, O h SH , Pi L, H atch H M , Shupe $\mathrm{T}$, Petersen $\mathrm{BE}$ : Teratoma formation leads to failure of treatment for Typel diabetes using embryonic stem cell-derived insulinproducing cells. Am. J. Pathol. 166(6), 1781-1791 (2005).

12. Costa M, D ottori M, N g E et al.: ThehESC line Envy expresses high levels of GFP in all 
differentiated progeny. $\mathrm{N}$ at. M ethods 2(4), 259-260 (2005).

13. Cao Y, Croll TI, Cooper-White J,, 0 'C onnor AJ, Stevens GW: Production and surface modification of polylactidebased polymeric scaffolds for soft tissue engineering. In: M ethods in Tissue Engineering. $\mathrm{H}$ atton $\mathrm{PV}$ (Ed.). H umana Press, Totowa, N J, USA 400 (2004).

14. Sidhu KS, Tuch BE: D erivation of three clones from human embryonic stem cell lines by FACS sorting and their characterization. Stem Cells D ev. 15(1), 61-69 (2006).

15. M ovassat J, Beattie GM , Lopez AD, $H$ ayek $A$ : Exendin 4 up-regulates expression of PDX 1 and hastens differentiation and maturation of human fetal pancreatic cells. J. Clin. Endocrinol. M etab. 87(10), 4775-4781 (2002).

16. Vallier L, Reynolds D, Pedersen RA: N odal inhibits differentiation of human embryonic stem cells along the neuroectodermal default pathway. $\mathrm{D}$ ev. Biol. 275(2), 403-421 (2004).

17. $M$ icallef $S$ J, Janes $M E, K$ nezevic $K$, $D$ avis RP, Elefanty AG, Stanley EG : Retinoic acid induces Pdx1-positive endoderm in differentiating mouse embryonic stem cells. D iabetes 54(2), 301-305 (2005).

18. Bai L, M eredith $G$, Tuch BE: Glucagonlike peptide-1 enhances production of insulin in insulin-producing cells derived from mouse embryonic stem cells. J. Endocrinol. 186(2), 343-352 (2005).
19. Jiang $F X, H$ arrison LC: Laminin-1 and epidermal growth factor family members co-stimulate fetal pancreas cell proliferation and colony formation. Differentiation 73(1), 45-49 (2005).

20. Badylak SF: The extracellular matrix as a scaffold for tissue reconstruction. Semin. Cell D ev. Biol. 13(5), 377-383 (2002)

21. Khoo M L, M CQ uade LR, Smith M S, Lees JG, Sidhu KS, Tuch BE: Growth and differentiation of embryoid bodies derived from human embryonic stem cells: effect of glucose and basic fibroblast growth factor. Biol. Reprod. 73(6), 1147-1156 (2005).

22. N elson CM, Tien J: M icrostructured extracellular matrices in tissue engineering and development. Curr. O pin. Biotechnol. 17(5), 518-523 (2006).

23. Eventov-Friedman $\mathrm{S}, \mathrm{K}$ atchman $\mathrm{H}$, Shezen $\mathrm{E}$ et al.: Embryonic pig liver, pancreas, and lung as a source for transplantation: optimal organogenesis without teratoma depends on distinct time windows. Proc. N atl Acad. Sci. USA 102(8), 2928-2933 (2005).

24. Hebrok M, Kim SK, M elton D A: $N$ otochord repression of endodermal Sonic hedgehog permits pancreas development. Genes D ev. 12(11), 1705-1713 (1998).

25. Beattie GM , Lopez AD, Bucay $N$ et al.: Activin A maintains pluripotency of human embryonic stem cells in the absence of feeder layers. Stem Cells 23(4), 489-495 (2005).

26. Kubo A, Shinozaki K, Shannon JM et al.: $D$ evelopment of definitive endoderm from embryonic stem cells in culture. D evelopment 131(7), 1651-1662 (2004).
27. Xu C, Rosler E, Jiang J et al.: Basic fibroblast growth factor supports undifferentiated human embryonic stem cell growth without conditioned medium. Stem Cells 23(3), 315-323 (2005).

28. Xu C, Inokuma M S, D enham J et al.: Feeder-free growth of undifferentiated human embryonic stem cells. $N$ at. Biotechnol. 19(10), 971-974 (2001).

29. Cooke M J, Stojkovic M, Przyborski SA: Growth of teratomas derived from human pluripotent stem cells is influenced by the graft site. Stem Cells D ev. 15(2), 254-259 (2006).

\section{Websites}

101. Invitrogen ${ }^{T M}$ www.invitrogen.com

102. ES Cell International www.escellinternational.com

103. BD Biosciences www.bdbiosciences.com

104. $R \& D$ Systems www.rndsystems.com

105. QIAGEN www1.qiagen.com

106. ABgene www. abgene.com

107. BIOCARE MEDICAL LLC www.biocare.net

108. Chemicon (now part of M illipore) www.chemicon.com

109. Sigma-Aldrich, Inc. www.Sigma-Aldrich.com

110. Santa Cruz Biotechnology, Inc. www.scbt.com

111. Emitech Ltd www.emitech.co.uk 